\title{
A Day-Ahead Generation Scheduling with Demand Response Considering Thermal Cycling Ramp
}

\author{
Wen-Shan Tan and Md Pauzi Abdullah \\ Centre of Electrical Energy Systems \\ Institute of Future Energy \\ Faculty of Electrical Engineering \\ Universiti Teknologi Malaysia \\ 81310, Johor Bahru, Malaysia
}

\author{
Mohamed Shaaban \\ Dept. of Electrical \& Electronic Engineering \\ Faculty of Engineering \\ Universiti Malaysia Sarawak \\ 94300 Kota Samarahan, Sarawak, Malaysia
}

\begin{abstract}
While increasing the shares of renewables is necessary for the transition to fossil-free energy provision, new challenges of flexibility are associated therein. Consumer flexibility is crucial to accommodate variable generation and peak loads. This paper proposes a chance-constrained programming (CCP) based day-ahead generation scheduling, including variable wind generation while engaging demand response (DR). Cycling ramp cost is introduced in this paper as a penalty in the objective cost function, to model the operation and maintenance $(O \& M)$ costs, which lead to the reduction of the wear and tear of the generator. The cycling of conventional generation and the dispatch of adjustable load are utilized to enhance the load following and net load flattening capabilities. The chance constraint is then converted into an equivalent mixed-integer linear programming (MILP) expression by using the Projected Disjunctive Reformulation (PDR), to speed up the computational performance. Test results on a 6-bus system show the merits of the proposed scheduling model.
\end{abstract}

Keywords-Unit commitment; generation unit ramping cost; storage device; renewable energy integration; mixed-integer linear programming

\section{NOMENCLATURE}

\section{A. Functions}

$C_{i, t}^{g} \quad$ Total generation cost of unit $i$ in time $t$

$C_{i, t}^{s u(s d)} \quad$ Total startup (shutdown) cost of unit $i$ in time $t$

$C_{i, t}^{r} \quad$ Total cycling ramp cost of unit $i$ in time $t$

$C G S_{b, t} \quad$ DR customer gross surplus at bus $b$ in time $t$

\section{B. $\quad$ Parameters}

$c_{f}^{\text {frus (frds) }}$ Price of step $f$ of up (down) flexible ramp surplus award [\$/MWh]

$d_{b, t}^{0} \quad$ inelastic demand at bus $b$ in time $t$ [MW]

$\bar{d}_{t} \quad$ Expected load demand [MW]

$d_{s, t}^{\text {net }} \quad$ Net load discrete realization level $s$ in time $t$ [MW]

$d r_{b, t}^{\max (\min ), c}$ Maximum (minimum) load curtailment at bus $b$ in time $t[\mathrm{MW}]$

This work was supported by the Malaysian Ministry of Education (MOE), under the Research University Grant (GUP), Vote No. 17H32, and in part by the Universiti Malaysia Sarawak (UNIMAS) via Special Grant Scheme, Grant No. F02/SpGS/1544/2017. $d r_{b, t}^{\max (\min ), s}$ Maximum (minimum) load shifting at bus $b$ in time $t$ [MW]

$D R C^{\max }$ Maximum allowable total load curtailment

$d r r_{b, t} \quad$ pick-up/drop-off rate of elastic demand at bus $b$ in time $t[\mathrm{MWh}]$

$h r_{i}^{g} \quad$ Incremental heat rate of unit $i[\mathrm{Btu} / \mathrm{kWh}]$

$p_{i}^{\max (\min )} \quad \mathrm{Max}(\mathrm{min})$ generation of unit $i$ [MW]

$S U(S D) \quad$ Start-up (shut-down) of step $l[\$]$

$s r_{t} \quad$ Spinning reserve in time $t$ [MW]

$\varepsilon^{u p(d n)} \quad \mathrm{Up}$ (down) flexible ramp surplus confidence level

$\pi_{i}^{g} \quad$ Incremental fuel cost of unit $i$ [\$/Btu]

$\lambda_{n, b, t} \quad$ Bidding price of block $n$ at bus $b$ in time $t$ [\$/MW]

$\chi_{n, b, t}^{d r} \quad$ Elastic demand of block $n$ at bus $b$ in time $t$ [MW]

C. Binary variables in time $t$

$u_{i(b), t} \quad$ On/off status of units $i$ (Curtail/Shifting status at bus $b$ )

$z_{s, t} \quad$ Probabilistic auxiliary variable of level $s$

$y_{h, t} \quad$ Probabilistic auxiliary variable of level $h$

D. $\quad$ Continuous variables at time $t$

$C R U_{i, t} \quad$ Up cycling ramp award of unit $i$ [MW]

$C R D_{i, t} \quad$ Up cycling ramp award of unit $i$ [MW]

$d r_{b, t}^{c} \quad$ Curtailable demand at bus $b$ in time $t$ [MW]

$d r_{b, t}^{s} \quad$ Shift-able demand at bus $b$ in time $t$ [MW]

$\bar{d}_{t}^{\text {net }} \quad$ Expected net load [MW]

$F C U_{i, t} \quad$ Up flexibility award of unit $i$ [MW]

$F C D_{i, t} \quad$ Down flexibility award of unit $i$ [MW]

$g_{j, t} \quad$ PDR auxiliary variable

$p_{i, t} \quad$ Dispatch of unit $i$ [MW]

$p r_{s, t} \quad$ Probability of net load level $s$

$r_{i, t}^{u p(d n)} \quad$ Ramp up (down) rate limit of unit $i$ [MWh]

D. $\quad$ Matrices and vectors

$S F \quad$ Shift factor

$P L^{\max } \quad$ Vector of upper limit for power flow 